\title{
A Sequential Algorithm for the Rigorous Design of Thermally Coupled Distillation Sequences
}

\author{
José A.Caballero, ${ }^{\mathrm{a}^{*}}$ Juan A. Reyes-Labarta, ${ }^{\mathrm{a}}$ Ignacio E. Grossmann. ${ }^{\mathrm{b}}$ \\ ${ }^{a}$ Department of Chemical Engineering, University of Alicante, Ap. Correos 99. \\ 03080,Alicante, Spain \\ ${ }^{b}$ Department of Chemical Engineering,Carnegie Mellon University, 5000 Forbes Av. \\ Pittsburgh, PA. 15213,USA. \\ caballer@ua.es
}

\begin{abstract}
A sequential design method is presented for the design of thermally coupled distillation sequences. The algorithm starts by selecting a set of sequences in the space of basic configurations in which the internal structure of condensers and reboilers is explicitly taken into account and extended with the possibility of including divided wall columns (DWC). This first stage is based on separation tasks (except by the DWCs) and therefore it does not provide an actual sequence of columns. In the second stage the best arrangement in $\mathrm{N}-1$ actual columns is performed taking into account operability and mechanical constraints. Finally, for a set of candidate sequences the algorithm try to reduce the number of total columns by considering Kaibel columns, elimination of transfer blocks or columns with vertical partitions. An example illustrate the different steps of the sequential algorithm
\end{abstract}

Keywords: Distillation, Thermally Coupled Distillation, MINLP, Process Integration, Process intensification.

\section{Introduction}

Thermally Coupled Distillation (TCD) has acquired a renewed interest because, when compared to conventional systems, it is possible to reach over $30 \%$ in energy reduction. Besides, if the search space includes Divided Wall Columns (DWC) important investment savings can also be achieved.

One of the major difficulties in the synthesis involving TCD is that the number of alternatives grows up much faster than when only conventional columns are considered. i.e. for a 5 component mixture there are 203 basic configurations (Giridhar \& Agrawal, 2010) if we consider also the internal structure of heat exchangers there are around $10^{4}$ alternatives, and if we consider the thermodynamically equivalent configurations the number of alternatives is around $2 \cdot 10^{5}$. (Caballero \& Grossmann, 2003).

In this work we propose a sequential algorithm, that combines shortcut models (based on Fenske - Underwood -Gilliland (FUG) methods) and rigorous process simulators to the rigorous design of complex TCD sequences. A description of the different stages is as follows:

1. Search in the space of basic configurations (Giridhar \& Agrawal, 2010) augmented with the internal structure of heat exchangers (Caballero \& Grossmann, 2006). Even 
though, consider only the basic configurations, and then optimize the internal structure of heat exchangers usually produce good results, Caballero and Grossmann (2013) showed that the optimal sequence of separation tasks can be lost in a sequential approach. Besides, those authors also showed that DWCs could have an important impact on the total cost of a given sequence and therefore it is convenient explicitly include DWCs in this stage. However, it is important that thermodynamically equivalent alternatives are not taken into account at this stage. Thermodynamically equivalent alternatives share the same sequence of separation tasks and, in most cases; the differences in cost are negligible. Therefore, except for the DWCs, we use an approach based on separation tasks instead of final columns. The final model is a MINLP model. A detailed description of this approach can be found in Caballero and Grossman (2013). Note that the sequence of task must be rearranged in actual columns (see points 3 and 4), that could have a considerable impact on the final cost especially if a configuration with less than $\mathrm{N}-1$ columns is generated.

Besides, in TCD it is common to find several solutions near the optimal one, which must be also taken into account. As a consequence we calculate all the sequences of separation tasks inside a given tolerance, say inside the $10 \%$ of the best one.

2. FUG equations assume constant volatilities and constant molar overflow. In actual systems these assumptions not always hold either in a single separation tasks or in different separations. The accumulation of errors could produce inaccurate results. A rigorous simulation (i.e. Aspen-Hysys) allows checking the model and, if necessary, we modify relative volatilities for each separation tasks until we get a good agreement between both models.

3. Selection of the best sequence of columns with exactly $\mathrm{N}-1$ columns ( $\mathrm{N}$ is the number of key components to be separated). At this point we must take into account two considerations. 1. A thermal couple -two streams, a liquid and a vapour connecting two sections- could induce sections with important differences in diameter. Although it is possible to build columns with different diameters, a column with three or four different diameters did not seem, at least practical. 2. Agrawal and Fidkowski (1998) showed that the sections must be arranged in actual columns in such a way that the vapour flows from higher to lower pressures. The vapour flow between columns can be controlled by a valve. Caballero \& Grossmann (2003) showed that for a given feasible sequence of separations tasks it is always possible to get that configuration using N-1 columns. Therefore, in this stage we search for the minimum cost feasible sequence formed by N-1 columns with a single diameter in which the vapour flows from higher to lower pressures. This usually means increasing the diameter of some sections, with its corresponding cost penalty. The feasibility must be checked to ensure that modifications in diameters do not create operability problems (i.e. weeping, flooding, etc.).

4. Selection of the best sequence with less than N-1 columns (Intensification). Some basic sequences of separation tasks allow reducing the number of columns to less than N-1 with a small or moderate penalty in energy consumption. There is a tradeoff between the reduction in the investment cost and the increase in the energy consumption. It would be possible to generate all the possible sequences with «reduced number» of columns (Shenvi et al, 2012), and try to select the best one. However a more practical approach consists of studying only the set of best 
sequences generated in step 1. If the maximum reduction in investment cost identifies a possible optimal sequence then the intensified sequence is rigorously calculated. Three possible intensification alternatives are taken into account: 1) Kaibel columns (Kaibel, 1984) -separate four key components using a DWC-. 2) Elimination of transfer blocks (Rong and Massimiliano, 2012). These blocks appear in sequences which have columns formed by a single section connecting other two columns. 3) Columns that can be merged in a single shell to form «columns with vertical partitions» (Agrawal, 2001). Or any combination of those.

It is also possible to reduce the number of columns by extracting some products as side streams, but in this case high purity requirements are difficult to get. But it must be taken into account if no high recoveries are necessary.

There are other modifications that could facilitate the operability of the sequence and can be taken into account. DWCs or Kaibel columns can be thermally coupled with the rest of the sequence, which could produce difficulties in the operation. In those cases it is convenient 'decouple' those columns by forcing the existence of a reboiler and a condenser. Shah and Agrawal (2010) suggested that some bi-directional flows (a liquid and a vapour stream exiting from some point in the middle of a column and entering in the middle of another column) can be substituted by a single liquid stream with a small energy penalty, but improving the operability of the column.

Finally, the existence of thermal couples makes that some separation tasks are working far away of their optimal conditions (conditions in which separation task would be operated if there were no interaction with other tasks). That excess of vapour (liquid) in some sections could be use as heating (cooling) media in other parts of the plant (Navarro et al, 2013). But of course, this analysis can be performed only taking into account the whole plant. Alternatively, if the excess of flow is too large (with also large diameters in the actual column) we should consider the possibility of include side heat exchangers. A comprehensive discussion can be found in the work by Navarro et al (2013)

We illustrate all these points with the following example.

\section{Example}

Consider a mixture of four lineal hydrocarbons; from n-hexane to n-nonane (All relevant data are shown in Table 1).

Table 1. Data for the example.( Rest of physical data from Aspen-Hysys TM database)

\begin{tabular}{lc|ll}
\hline Components & $\begin{array}{c}\text { Feed flow } \\
(\mathrm{kmol} / \mathrm{h})\end{array}$ & & \\
\hline $\mathrm{A}=$ n-hexane & 120 & Pressure & $1.2 \mathrm{~atm}$ \\
$\mathrm{~B}=$ n-heptane & 90 & Feed liquid fraction & 1 \\
$\mathrm{C}=$ n-octane & 60 & Vapour Steam cost & $14.05 \$ / \mathrm{GJ}$ \\
$\mathrm{D}=$ n-nonane & 30 & Cooling water cost & $0.354 \$$ GJ \\
& & Cost data source & Turton et al (2013) \\
\hline
\end{tabular}

The following constraints have been added to the model in order to generate nondifficult to operate sequences: 1) Divided wall columns must have a condenser and a reboiler. In other words, they cannot be thermally coupled with the rest of the system. 2) The flow transfer between two columns using side streams is forced to be saturated 
liquid. 3) We search for actual columns with a single diameter, and configurations for which it is possible to establish a gradient of pressures in such a way that the vapour flows from higher to lower pressures. 4) We consider only columns with a single internal wall.

As commented before we obtained not only the best solution but a set of solutions inside a given tolerance. For this example there are 6 different solutions with a TAC that differ in less than $10 \%$. The best solution obtained corresponds to a fully thermally couple configuration (extended Petlyuk arrangement) with a total annualized cost (TAC) equals to $2261.9 \mathrm{k} \$ / \mathrm{y}$. If we rearrange the sequence of tasks in actual columns there is an increment of cost, because the increase in the diameter of some sections, to $2336.7 \mathrm{k} \$ / \mathrm{y}$. (around 3.3\%). Figure 1 shows the best solution of stage 1 after the rearrangement in actual columns.

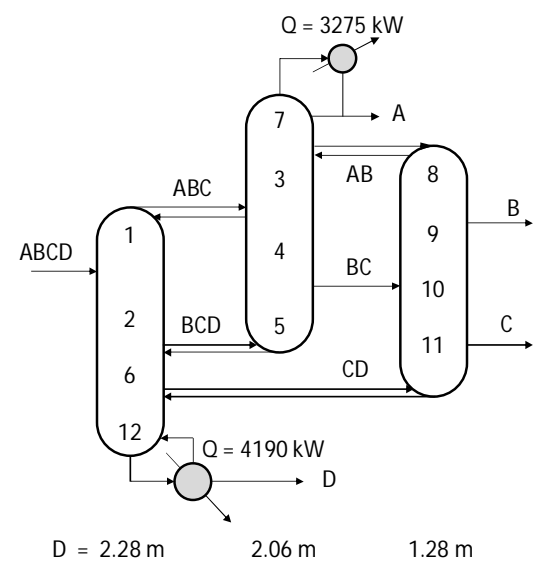

Figure 1. Best solution after steps 1 and 2
A Kaibel sequence can be generated from a fully TCD sequence by removing intermediate mixtures that do not include extreme volatility components ( $\mathrm{BC}$ in this case). If the energy penalty is not too large the reduction in the number of shells could become the Kaibel column the optimal one. In this example, a simple calculation at minimum reflux conditions shows that there is an energy penalty around $18.5 \%$. Taking into account energy consumption is around the $70 \%$ of the TAC we can discard the Kaibel column.

The first, second, third and fourth solutions share the same sequence of separation tasks, and differs each other only in some heat exchangers, therefore we skip here these alternatives (second solution includes a reboiler in the separation $\mathrm{BC} / \mathrm{CD}$; the third one includes reboilers in separation tasks $\mathrm{ABC} / \mathrm{BCD}$ and $\mathrm{BC} / \mathrm{CD}$; and the fourth solution a reboiler in $\mathrm{ABC} / \mathrm{BCD})$.

Especial mention deserves the fifth solution. For this solution the TAC increases to $2455 \mathrm{k} \$ / \mathrm{y}$ (around $8 \%$ higher). In this case it is possible to identify a 'transfer block', which allows reducing by one the number of columns, with almost no penalty in energy consumption, (see Figure 2). We can go a step forward and merge these two columns in a single divided wall column. Remark also that in the final configuration the areas of each section do not need to be modified!

The TAC for the sequence in Figure 2.d is reduced to $2276 \mathrm{k} \$ / \mathrm{y}$. Therefore, the intensified configuration in Figure $2 \mathrm{~d}$ has the same TAC than the best sequence formed by $\mathrm{N}-1$ columns (difference around $1 \%$ ). The final decision on what sequence to build must be based in other kind of considerations. 
It is worth remarking that the first sequence with a DWC has a TAC equal to 2821.3 $\mathrm{k} \$ / \mathrm{y}$ (around $25 \%$ higher). This is due to the fact that we have forced the DWC not to be coupled with the rest of the system. In other words, the DWC must have a condenser and a reboiler (see Figure 3a). Intermediate heat exchangers could have an important impact on the total cost. For example, if for the best solution we force a condenser in the separation task $\mathrm{AB} / \mathrm{BC}$ (this heat exchanger does not appear in any of the best solutions) the cost increases almost in a $30 \%(2910 \mathrm{k} \$ / \mathrm{y}$.)
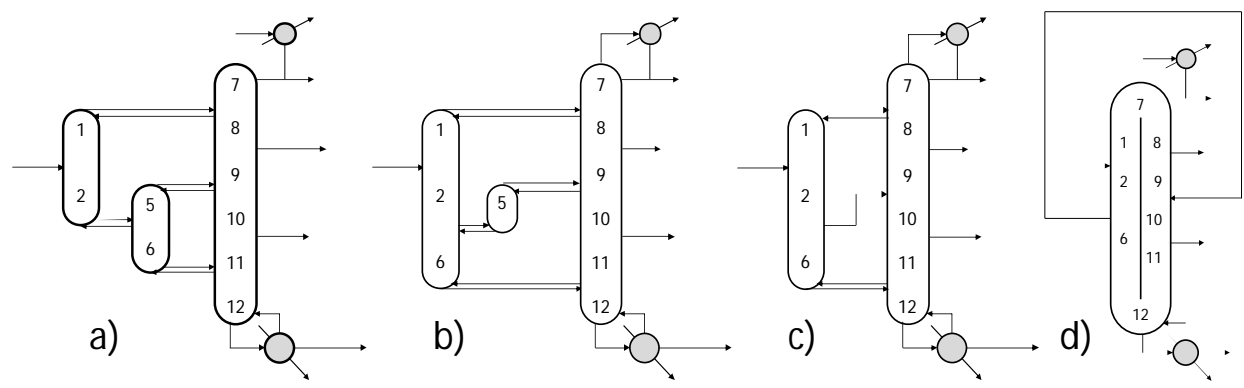

Figure 2. Process intensification for the $5^{\text {th }}$ sequence. a) A feasible arrangement in actual columns. b) A thermodynamically equivalent configuration with a «transfer block». c) Configuration removing section 5. d) Divided wall column thermodynamically equivalent to configuration $\mathrm{c}$.

If we allow the DWCs to be coupled with the rest of the system then the optimal solution is a fully TCD with an internal wall (See Figure $3 b$ ).
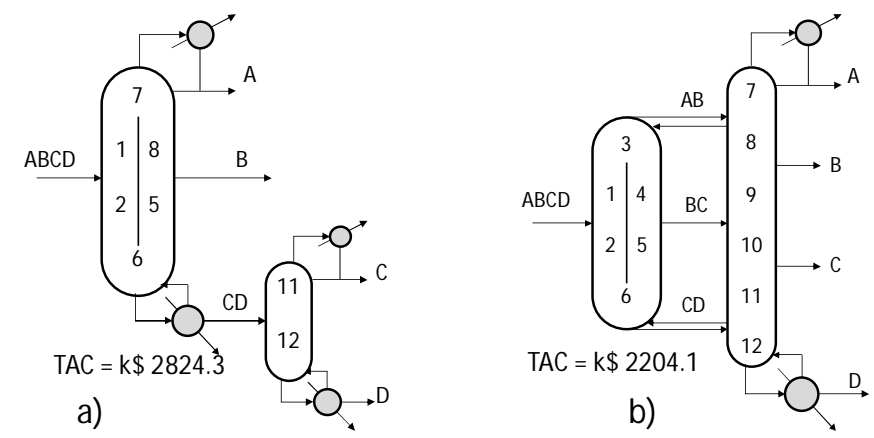

Figure 3. a) Best solution with a divided wall in which the DWC is forced to have reboiler and condenser. b) Best solution if DWCs can be thermally coupled.

\section{Conclusions}

A systematic procedure for the synthesis and intensification of column configurations for multicomponent distillation is presented. The algorithm starts by searching in the space of basic configurations extended with the internal structure of heat exchangers (reboilers and condensers do not associated to final products). It has been shown that some internal heat exchangers can have a large impact on the performance of the complete system. If this internal structure of heat exchangers is not taken into account there is a large probability of losing the optimal solution. On the other side it is not convenient to explicitly consider, at this stage, the final arrangement in actual columns. This is because there are a potential very large number of thermodynamically equivalent 
configurations with similar cost. So a good approach is to use a task based instead of a column based approach.

The optimal sequence of tasks must be rearranged in actual columns. To that end the algorithm takes into account operability constraints and some necessary modification in the diameter of some column sections to avoid building columns with three or more different diameters. Other considerations like limiting the total length can be addressed either by increasing the number of columns or modifying the internals in the column (i.e. tray separation). However, all these modifications must be carefully checked to avoid column malfunctions.

Finally, it is possible try to intensify the total system by reducing the number of column shells. Different alternatives where considered: Kaibel columns, elimination of transfer blocks and merge columns using vertical partitions. However, except in some DWCs, the reduction in the number columns involves a penalty in energy and as a consequence the trade-offs must be carefully studied. But the example shows that these alternatives must be considered

\section{Acknowledgements.}

The authors wish to acknowledge the financial support by the Ministry of Economy and Competitiveness from Spain, under the project CTQ 2012-37039-C02-02.

\section{References}

R. Agrawal, 2001. Multicomponent distillation columns with partitions and multiple reboilers and condensers. Industrial \& Engineering Chemistry Research 40(20) 4258-4266.

R. Agrawal, Z. T. Fidkowski, 1998. More operable arrangements of fully thermally coupled distillation columns. AIChE Journal 44(11) 2565-2568.

J. A Caballero, I. E. Grossmann, 2003. Thermodynamically Equivalent Configurations for Thermally Coupled Distillation. AIChE Journal, 49(11) 2864-2884.

J. A. Caballero, I. E. Grossmann, 2006. Structural considerations and modeling in the synthesis of heat-integrated-thermally coupled distillation sequences. Industrial and Engineering Chemistry Research 45(25) 8454-8474.

J. A. Caballero, I. E. Grossmann, 2013. Synthesis of complex thermally coupled distillation systems including divided wall columns. AIChE Journal 59(4) 1139-1159.

A. Giridhar, R. Agrawal, 2010. Synthesis of distillation configurations: I. Characteristics of a good search space. Computers \& Chemical Engineering, 34(1) 73-83.

G. Kaibel, 1987. Distillation Columns with Vertical Partitions. Chemical Engineering Techology 10(1) 92-98.

M A. Navarro-Amoros, R. Ruiz-Femenia, J. A. Caballero, 2013. A new technique for recovering energy in thermally coupled distillation using vapor recompression cycles." AIChE Journal 59(10) 3767-3781.

B. G. Rong, M. Errico, 2012. Synthesis of intensified simple column configurations for multicomponent distillations. Chemical Engineering and Processing: Process Intensification $62,1-17$.

V. H. Shah, R. Agrawal, 2010. Are All Thermal Coupling Links between Multicomponent Distillation Columns Useful from an Energy Perspective? Industrial \& Engineering Chemistry Research 50(3) 1770-1777.

A. A. Shenvi, ,V.H. Shah, J. A. Zeller, R. Agrawal, 2012. A synthesis method for multicomponent distillation sequences with fewer columns. AIChE Journal 58(8): 2479-2494.

R. Turton, R. Bailei, R. C., Whiting, B. Wallace, J. A. Shaeiwitz, D. Bhattacharyya. 2013. Analysis, Synthesis and Design of Chemical Processes. 4th Edition. Upper Saddle River, NJ. USA., Pearson Education, Inc. 